\title{
EXPONENTIAL STABILITY IN A SCALAR FUNCTIONAL DIFFERENTIAL EQUATION
}

EDUARDO LIZ AND MIHÁLY PITUK

Received 21 March 2006; Revised 16 August 2006; Accepted 21 September 2006

We establish a criterion for the global exponential stability of the zero solution of the scalar retarded functional differential equation $x^{\prime}(t)=L\left(x_{t}\right)+g\left(t, x_{t}\right)$ whose linear part $y^{\prime}(t)=L\left(y_{t}\right)$ generates a monotone semiflow on the phase space $C=C([-r, 0], \mathbb{R})$ with respect to the exponential ordering, and the nonlinearity $g$ has at most linear growth.

Copyright (c) 2006 E. Liz and M. Pituk. This is an open access article distributed under the Creative Commons Attribution License, which permits unrestricted use, distribution, and reproduction in any medium, provided the original work is properly cited.

\section{Introduction}

Given $r \in \mathbb{R}^{+}=[0, \infty)$, let $C=C([-r, 0], \mathbb{R})$ denote the Banach space of continuous functions mapping $[-r, 0]$ into $\mathbb{R}$ equipped with the supremum norm

$$
\|\phi\|=\sup _{-r \leq \theta \leq 0}|\phi(\theta)|, \quad \phi \in C
$$

In this paper, we are concerned with the stability properties of the zero solution of the scalar retarded functional differential equation

$$
x^{\prime}(t)=L\left(x_{t}\right)+g\left(t, x_{t}\right)
$$

where $L: C \rightarrow \mathbb{R}$ is a bounded linear functional and the function $g: \mathbb{R}^{+} \times C \rightarrow \mathbb{R}$ is continuous and has at most linear growth in the sense that for some $\gamma \geq 0$, we have that

$$
|g(t, \phi)| \leq \gamma\|\phi\|, \quad t \in \mathbb{R}^{+}, \phi \in C .
$$

As usual, the symbol $x_{t} \in C$ is defined by $x_{t}(\theta)=x(t+\theta)$ for $-r \leq \theta \leq 0$. 
With (1.2), we can associate an initial condition of the form

$$
x_{\sigma}=\phi
$$

where $\sigma \in \mathbb{R}^{+}$and $\phi \in C$.

Equation (1.2) includes as a special case the equation

$$
x^{\prime}(t)=-d x(t-\tau)+f(x(t-\omega))
$$

where $\tau, \omega \in \mathbb{R}^{+}, d \in \mathbb{R}$, and $f: \mathbb{R} \rightarrow \mathbb{R}$ is continuous. The list of the above symbols is the following:

$$
\begin{gathered}
r=\max \{\tau, \omega\}, \\
L(\phi)=-d \phi(-\tau), \quad \phi \in C, \\
g(t, \phi)=f(\phi(-\omega)), \quad t \in \mathbb{R}^{+}, \phi \in C .
\end{gathered}
$$

Equation (1.5) was studied by Gyóri [6], who established the following criterion for the global attractivity of the zero solution of (1.5).

Theorem 1.1 [6, Theorem 4.2]. Suppose that

$$
\begin{gathered}
d>0, \quad d \tau<\frac{1}{e}, \\
x f(x) \geq 0, \quad x \in \mathbb{R} .
\end{gathered}
$$

Then all solutions of (1.5) tend to zero if and only if

$$
|f(x)|<d|x|, \quad x \in \mathbb{R} \backslash\{0\} .
$$

In this paper, among others, we will show that if hypothesis (1.9) of Theorem 1.1 is replaced with the slightly stronger condition

$$
\sup _{x \in \mathbb{R} \backslash\{0\}} \frac{|f(x)|}{|x|}<d,
$$

then the zero solution of (1.5) is exponentially stable. Recall that the zero solution of (1.2) is (globally uniformly) exponentially stable if there exist constants $M>1$ and $\lambda<0$ such that if $x$ is a noncontinuable solution of (1.2) with initial value (1.4) for some $\sigma \in \mathbb{R}^{+}$ and $\phi \in C$, then $x$ is defined on $[\sigma-r, \infty)$ and satisfies the inequality

$$
|x(t)| \leq M\|\phi\| e^{\lambda(t-\sigma)}, \quad t \geq \sigma .
$$


The following theorem is a simple corollary of our more general result presented in Section 3 (see Theorem 3.1).

Theorem 1.2. Suppose conditions (1.7) and (1.10) hold. Then the zero solution of (1.5) is exponentially stable.

The proof of Theorem 1.1 by Győri [6] is based on the variation-of-constants formula and the fact that under condition (1.7), the fundamental solution of the linear part of (1.5),

$$
y^{\prime}(t)=-d y(t-\tau)
$$

is nonnegative. Our approach combines Györi's idea with the observation made by Smith and Thieme [10]. Namely, if (1.7) holds, then the linear equation (1.12) generates a monotone semiflow on $C$ with respect to the exponential ordering. An important tool in our proof is a useful inequality between the solutions of the linear part of (1.2) (see Lemma 2.2).

It should be mentioned that similar techniques and ideas were used earlier by the participants of the Perm Seminar on Functional Differential Equations (Perm, Russia). For details, see $[1,5]$ and the references therein. For recent stability criteria which are relevant to our study, the reader is referred to the papers $[2,3]$.

The paper is organized as follows. In Section 2, we summarize some facts from the theory of functional differential equations generating a monotone semiflow and we establish some auxiliary results which will be used in our proof. The main theorem and its proof are given in Section 3.

\section{Preliminaries}

Consider the linear autonomous functional differential equation

$$
y^{\prime}(t)=L\left(y_{t}\right)
$$

with $L$ as in (1.2). It is well known [7] that if $(\sigma, \phi) \in \mathbb{R}^{+} \times C$, then the unique (noncontinuable) solution $y$ of (2.1) with initial value $y_{\sigma}=\phi$ exists for all $t \geq \sigma$. Denote it by $y(t)=y(t ; \sigma, \phi)$ or $y_{t}=y_{t}(\sigma, \phi)$ depending on whether we interpret the solution $y$ in $\mathbb{R}$ or in $C$.

Let us summarize some facts from the theory of monotone dynamical systems. For proofs and more details, see [9, Chapter 6]. If $\mu>0$, then the exponential ordering $\leq_{\mu}$ is the partial order in $C$ induced by the convex closed cone

$$
K_{\mu}=\left\{\phi \in C \mid \phi \geq 0, \phi(\theta) e^{\mu \theta} \text { is nondecreasing on }[-r, 0]\right\} .
$$

Thus, $\phi \leq_{\mu} \psi$ for some $\phi, \psi \in C$ if and only if $\psi-\phi \in K_{\mu}$. We write $\phi<_{\mu} \psi$ if $\phi \leq_{\mu} \psi$ and $\phi \neq \psi$.

Equation (2.1) generates a continuous global semiflow on $C$ by

$$
t \longmapsto y_{t}(0, \phi), \quad t \in \mathbb{R}^{+}, \phi \in C
$$


In $[9$, Chapter 6 , Theorem 1.1], it is shown that the above semiflow is monotone with respect to the ordering $\leq_{\mu}$, that is,

$$
\phi \leq_{\mu} \psi \quad \text { implies } y_{t}(0, \phi) \leq_{\mu} y_{t}(0, \psi), \quad \forall t \in \mathbb{R}^{+},
$$

if and only if

$$
L(\phi)+\mu \phi(0) \geq 0 \quad \text { whenever } \phi \in C, \phi \geq_{\mu} 0 .
$$

Throughout the paper, instead of (2.5), we will assume the slightly stronger condition

$$
L(\phi)+\mu \phi(0)>0 \quad \text { whenever } \phi \in C, \phi>_{\mu} 0 .
$$

As shown in [9, Chapter 6, Theorem 2.3], condition (2.6) implies that the semiflow (2.3) is strongly order-preserving (see [9, Chapter 1] for the definition of a strongly orderpreserving semiflow which is not needed here). A sufficient condition for (2.6) to hold can be formulated in terms of the signed Borel measure representing the bounded linear functional $L$. If $L$ has the form

$$
L(\phi)=\int_{[-r, 0]} \phi(\theta) d \nu(\theta), \quad \phi \in C,
$$

where $v$ is a regular signed Borel measure, then condition (2.6) holds if

$$
\mu+\int_{H} e^{-\mu \theta} d \nu(\theta)>0
$$

for $H=[-r, 0]$ and $H=(\theta, 0]$ for each $\theta \in[-r, 0)$ (see [9, Chapter 6, Proposition 1.4 and Remark 2.2]). In the case of the simple equation (1.12), the linear functional $L(\phi)=$ $-d \phi(-\tau)$ is represented by the measure $\nu=-d \delta_{-r}$, where $\delta_{-r}$ is the Dirac measure with support on $\{-r\}$. Condition (2.8) reduces to

$$
\mu-d e^{\mu \tau}>0
$$

In most cases the particular value of $\mu>0$ is not of interest. It is routine to show that (2.9) holds for some $\mu>0$ if and only if

$$
d^{+} \tau<\frac{1}{e}, \quad d^{+}=\max \{0, d\}
$$

Consequently, under condition (1.7) of Theorem 1.1, the strong monotonicity condition (2.6) is satisfied for (1.12) for some $\mu>0$.

Recall that the fundamental solution $u$ of $(2.1)$ is the unique solution of $(2.1)$ on $[-r, \infty)$ with initial data

$$
u(\theta)= \begin{cases}0 & \text { for }-r \leq \theta<0 \\ 1 & \text { for } \theta=1\end{cases}
$$


The family of special solutions $y\left(\cdot ; 0, e_{\lambda}\right)$ of $(2.1)$, where $\lambda \in \mathbb{R}$ and $e_{\lambda} \in C$ is the exponential function

$$
e_{\lambda}(\theta)=e^{\lambda \theta}, \quad-r \leq \theta \leq 0,
$$

will play an important role in the sequel. For $\lambda=0$, write $y\left(\cdot ; 0, e_{0}\right)=v$ for brevity. Thus, $v$ is the unique solution of $(2.1)$ on $[-r, \infty)$ with initial values $v(\theta)=1$ identically for $\theta \in[-r, 0]$. In the following result, extracted from [8], we summarize some properties of the special solutions $u$ and $v$ of (2.1). It says that under the strong monotonicity condition (2.6), both solutions are positive for $t \in \mathbb{R}^{+}$and $v$ dominates all other solutions of (2.1).

Proposition 2.1 [8, Propositions 2.1 and 2.3]. Suppose that (2.6) holds for some $\mu>0$. Then

(i) the solutions $u$ and $v$ are positive on $[0, \infty)$,

(ii) there exists $K>1$ such that for all $\phi \in C$ and $t \geq-r$,

$$
|y(t ; 0, \phi)| \leq K\|\phi\| v(t) .
$$

The next lemma shows that in Proposition 2.1, the special solution $v$ can be replaced with any of the solutions $y\left(\cdot ; 0, e_{\lambda}\right)$, where $\lambda \in(-\mu, 0)$.

Lemma 2.2. Suppose that (2.6) holds for some $\mu>0$ and let $\lambda \in(-\mu, 0)$. Then

(i) the solution $y\left(\cdot ; 0, e_{\lambda}\right)$ of $(2.1)$ is positive on $[-r, \infty)$,

(ii) there exists $M>1$ such that for all $\phi \in C$ and $t \geq-r$,

$$
|y(t ; 0, \phi)| \leq M\|\phi\| y\left(t ; 0, e_{\lambda}\right)
$$

Proof. Let $\lambda \in(-\mu, 0)$. We claim that if $k \geq 1$ is sufficiently large, then

$$
e_{0} \leq_{\mu} k e_{\lambda}
$$

Indeed, by the definition of the ordering $\leq_{\mu},(2.15)$ is equivalent to

$$
1 \leq k e^{\lambda \theta}, \quad \frac{d}{d \theta}\left(e^{\mu \theta}\left(k e^{\lambda \theta}-1\right)\right)=e^{\mu \theta}\left(k(\mu+\lambda) e^{\lambda \theta}-\mu\right) \geq 0
$$

for all $\theta \in[-r, 0]$. Since $\mu+\lambda>0$, the last two conditions, and hence (2.15), certainly hold if $k \geq 1$ is sufficiently large. If $k \geq 1$ is chosen in this way, then the monotonicity conditions (2.4) and (2.15) imply that

$$
y_{t}\left(0, e_{0}\right) \leq_{\mu} y_{t}\left(0, k e_{\lambda}\right)=k y_{t}\left(0, e_{\lambda}\right), \quad t \in \mathbb{R}^{+} .
$$

In particular,

$$
v(t)=y\left(t ; 0, e_{0}\right) \leq k y\left(t ; 0, e_{\lambda}\right), \quad t \geq-r .
$$

Using the last inequality in (2.13), we obtain (2.14) with $M=k K$. Thus, conclusions (i) and (ii) of the lemma follow from Proposition 2.1. 
Consider the nonhomogeneous equation

$$
x^{\prime}(t)=L\left(x_{t}\right)+h(t)
$$

associated with (2.1), where $h:[\sigma, \infty) \rightarrow \mathbb{R}$ with $\sigma \in \mathbb{R}$ is continuous. By the variation-ofconstants formula (see [7, Chapter 6]), the unique solution $x$ of $(2.19)$ on $[\sigma-r, \infty)$ with initial value $x_{\sigma}=\phi$ is given by

$$
x(t)=y(t-\sigma ; 0, \phi)+\int_{\sigma}^{t} u(t-s) h(s) d s, \quad t \geq \sigma,
$$

where $y(\cdot ; 0, \phi)$ and $u$ are the solutions of the homogeneous equaion (2.1) defined as before.

We conclude this section with an integral identity involving the special solutions $u$ and $y\left(\cdot ; 0, e_{\lambda}\right)$ of $(2.1)$, where $\lambda \in \mathbb{R}$ is a root of the equation

$$
\Delta(\lambda)=0, \quad \Delta(\lambda)=\lambda-L\left(e_{\lambda}\right)-\gamma e^{-\lambda r} .
$$

Lemma 2.3. If $\lambda \in \mathbb{R}$ is a root of (2.21), then

$$
y\left(t-\sigma ; 0, e_{\lambda}\right)+\int_{\sigma}^{t} u(t-s) \gamma e^{\lambda(s-r-\sigma)} d s=e^{\lambda(t-\sigma)}
$$

for all $t \geq \sigma \geq 0$.

Proof. Clearly, if $\lambda \in \mathbb{R}$ is a root of (2.21), then the function $x(t)=e^{\lambda(t-\sigma)}$ is a solution of the equation

$$
x^{\prime}(t)=L\left(x_{t}\right)+\gamma x(t-r)
$$

with initial value $x_{\sigma}=e_{\lambda}$. The desired conclusion (2.22) follows from (2.20) by letting $h(t)=\gamma x(t-r)=\gamma e^{\lambda(t-r-\sigma)}$ for $t \geq \sigma$.

\section{The main result}

Our main result is the following theorem which provides a criterion for the exponential stability of the zero solution of (1.2) under the hypothesis that the linear functional $L$ satisfies the strong monotonicity condition (2.6) for some $\mu>0$. Sufficient conditions for (2.6) to hold were mentioned in Section 2 (see also [9, Chapter 6]).

Theorem 3.1. Suppose that there exist $\gamma \geq 0$ and $\mu>0$ such that (1.3) and (2.6) hold. Assume further that

$$
\gamma<-L\left(e_{0}\right)
$$

where $e_{0}(\theta)=1$ identically for $\theta \in[-r, 0]$. Then the zero solution of $(1.2)$ is exponentially stable. More precisely, if $\lambda$ is the unique root of $(2.21)$ in $(-\mu, 0)$ and the constant $M$ has the meaning from Lemma 2.2(ii), then any noncontinuable solution $x$ of (1.2) with initial value (1.4) for some $\sigma \in \mathbb{R}^{+}$and $\phi \in C$ is defined on $[\sigma-r, \infty)$ and (1.11) holds. 
Remark 3.2. The existence and uniqueness of $\lambda$ is part of the conclusion of the above theorem.

Proof. First we show the existence of a solution $\lambda$ of $(2.21)$ in the interval $(-\mu, 0)$. Since $e_{-\mu}>_{\mu} 0$, inequality (2.6) implies that $L\left(e_{-\mu}\right)+\mu>0$. This and (3.1) yield that $\Delta(-\mu)<0$ and $\Delta(0)>0$. Since $\Delta$ is continuous, the intermediate value theorem implies the existence of $\lambda \in(-\mu, 0)$ at which $\Delta(\lambda)=0$.

Let $\lambda$ be any root of $(2.21)$ in the interval $(-\mu, 0)$ and let $M$ have the meaning from Lemma 2.2(ii). We will show that the exponential estimate (1.11) holds for any noncontinuable solution $x$ of (1.2) with initial value (1.4). Let $x$ be a noncontinuable solution of the initial-value problem (1.2) and (1.4) for some $(\sigma, \phi) \in \mathbb{R}^{+} \times C$. Then $x$ is defined on some interval $[\sigma-r, b)$, where $\sigma<b \leq \infty$. Taking into account that $\lambda<0$, (1.4) implies for $t \in[\sigma-r, \sigma]$ that

$$
|x(t)| \leq\|\phi\| \leq\|\phi\| e^{\lambda(t-\sigma)} .
$$

From this and the fact that $M>1$, we obtain that for any $\epsilon>0$ and $t \in[\sigma-r, \sigma]$,

$$
|x(t)|<M(\|\phi\|+\epsilon) e^{\lambda(t-\sigma)}
$$

We claim that the strict inequality (3.3) is also valid for $t \in(\sigma, b)$. Otherwise, there exists $t_{1} \in(\sigma, b)$ such that

$$
\begin{gathered}
|x(t)|<M(\|\phi\|+\epsilon) e^{\lambda(t-\sigma)}, \quad \sigma-r \leq t<t_{1}, \\
\left|x\left(t_{1}\right)\right|=M(\|\phi\|+\epsilon) e^{\lambda\left(t_{1}-\sigma\right)} .
\end{gathered}
$$

Since $\lambda<0$, (3.4) yields

$$
\left\|x_{t}\right\|=\sup _{-r \leq \theta \leq 0}|x(t+\theta)| \leq M(\|\phi\|+\epsilon) e^{\lambda(t-r-\sigma)}, \quad \sigma \leq t<t_{1} .
$$

By the variation-of-constants formula (2.20), we have that

$$
x\left(t_{1}\right)=y\left(t_{1}-\sigma ; 0, \phi\right)+\int_{\sigma}^{t_{1}} u\left(t_{1}-s\right) g\left(s, x_{s}\right) d s .
$$

From this, using (1.3), the positivity of the fundamental solution $u$ on $\mathbb{R}^{+}$(see Proposition 2.1(i)), Lemma 2.2, and (3.6), we obtain

$$
\begin{aligned}
\left|x\left(t_{1}\right)\right| & \leq\left|y\left(t_{1}-\sigma ; 0, \phi\right)\right|+\int_{\sigma}^{t_{1}} u\left(t_{1}-s\right) \gamma\left\|x_{s}\right\| d s \\
& \leq M\|\phi\| y\left(t_{1}-\sigma ; 0, e_{\lambda}\right)+\int_{\sigma}^{t_{1}} u\left(t_{1}-s\right) \gamma M(\|\phi\|+\epsilon) e^{\lambda(s-r-\sigma)} d s \\
& <M(\|\phi\|+\epsilon)\left[y\left(t_{1}-\sigma ; 0, e_{\lambda}\right)+\int_{\sigma}^{t_{1}} u\left(t_{1}-s\right) \gamma e^{\lambda(s-r-\sigma)} d s\right] \\
& =M(\|\phi\|+\epsilon) e^{\lambda\left(t_{1}-\sigma\right)},
\end{aligned}
$$


the last equality being a consequence of the identity (2.22) of Lemma 2.3. This contradicts (3.5), and thus (3.3) holds for all $t \in[\sigma-r, b)$. Letting $\epsilon \rightarrow 0$ in (3.3), we obtain

$$
|x(t)| \leq M\|\phi\| e^{\lambda(t-\sigma)}, \quad \sigma \leq t<b .
$$

By a well known continuation theorem (see [7, Chapter 2, Theorem 3.2]), the last inequality implies that $b=\infty$, and thus (1.11) holds.

It remains to show the uniqueness of the solution $\lambda$ of $(2.21)$ in the interval $(-\mu, 0)$. Suppose by the way of contradiction that (2.21) has two solutions $\lambda_{1}, \lambda_{2} \in(-\mu, 0), \lambda_{1}<\lambda_{2}$. If we let

$$
g(t, \phi)=\gamma \phi(-r), \quad t \in \mathbb{R}^{+}, \phi \in C,
$$

then condition (1.3) is satisfied and (1.2) reduces to the linear equation (2.23) which has the solution $x(t)=e^{\lambda_{2}(t-\sigma)}$ with initial value $x_{\sigma}=e_{\lambda_{2}}$. Since $\lambda=\lambda_{1}$ is a root of (2.21) belonging to the interval $(-\mu, 0)$, according to the previous part of the proof, the above solution satisfies the exponential esimate (1.11), that is,

$$
e^{\lambda_{2}(t-\sigma)} \leq M \| e_{\lambda_{2}}|| e^{\lambda_{1}(t-\sigma)}, \quad t \geq \sigma
$$

Hence

$$
e^{\left(\lambda_{2}-\lambda_{1}\right) t} \leq M e^{-\lambda_{2} r} e^{\left(\lambda_{2}-\lambda_{1}\right) \sigma}, \quad t \geq \sigma
$$

a contradiction, since $e^{\left(\lambda_{2}-\lambda_{1}\right) t} \rightarrow \infty$ as $t \rightarrow \infty$.

As noted in Section 1, Theorem 1.2 is a simple consequence of Theorem 3.1. As another example, consider the equation

$$
x^{\prime}(t)=\sum_{i=1}^{\infty} a_{i} x\left(t-r_{i}\right)+K\left(x_{t}\right)
$$

where $a_{i} \in \mathbb{R}$ and $0 \leq r_{i} \leq r$ for some $r>0$ and $i=1,2, \ldots, \sum_{i=1}^{\infty}\left|a_{i}\right|<\infty$, and $K: C \rightarrow \mathbb{R}$ is a bounded linear functional. Equation (3.13) has recently been studied by Faria and Huang [4]. In [4, Example 3.1], it is shown that the zero solution of (3.13) is exponentially stable if

$$
\sum_{i=1}^{\infty}\left|a_{i}\right| e^{r_{i} / r}+\|K\| e<\frac{1}{r}, \quad \sum_{i=1}^{\infty} a_{i}+K\left(e_{0}\right)<0,
$$

where $\|K\|$ denotes the operator norm of $K$ and $e_{0} \in C$ has the same meaning as in (2.12). The following corollary of Theorem 3.1 is an improvement of the above result. 
Corollary 3.3. Any of the conditions (i), (ii), and (iii) below is sufficient for the exponential stability of the zero solution of (3.13):

(i) $\sum_{i=1}^{\infty} a_{i}^{-} e^{r_{i} / r}+\|K\| e<1 / r$ and $\sum_{i=1}^{\infty} a_{i}+K\left(e_{0}\right)<0$,

(ii) $\sum_{i=1}^{\infty} a_{i}^{-} e^{r_{i} / r}<1 / r$ and $\|K\|+\sum_{i=1}^{\infty} a_{i}<0$,

(iii) $\|K\| e<1 / r$ and $\sum_{i=1}^{\infty}\left|a_{i}\right|+K\left(e_{0}\right)<0$,

where $a_{i}^{-}=\max \left\{0,-a_{i}\right\}$ for $i=1,2, \ldots$.

Proof. The result follows from Theorem 3.1 when

$$
\begin{gathered}
L(\phi)=\sum_{i=1}^{\infty} a_{i} \phi\left(-r_{i}\right)+K(\phi), \quad \gamma=0, \\
L(\phi)=\sum_{i=1}^{\infty} a_{i} \phi\left(-r_{i}\right), \quad \gamma=\|K\|, \\
L(\phi)=K(\phi), \quad \gamma=\sum_{i=1}^{\infty}\left|a_{i}\right|,
\end{gathered}
$$

respectively. It is easily shown that under each of the conditions (i), (ii), and (iii) of the corollary, condition (2.8), and hence (2.6), is satisfied with $\mu=1 / r$ for the corresponding linear functional $L$.

\section{Acknowledgments}

This research was done within the framework of the Hungarian-Spanish Intergovernmental S\&T Cooperation Programme, supported by the Research and Technology Innovation Found and Foundation Mecenatúra, Grant no. E-8/04, Spanish reference: HH2004-0018. E. Liz was supported in part by MEC (Spain) and FEDER under grant MTM2004-06652C03-02. M. Pituk was supported in part by the Hungarian National Foundation for Scientific Research Grant no. T 046929. The authors wish to thank the referees for valuable comments.

\section{References}

[1] N. V. Azbelev and P. M. Simonov, Stability of Differential Equations with Aftereffect, Stability and Control: Theory, Methods and Applications, vol. 20, Taylor \& Francis, London, 2003.

[2] L. Berezansky and E. Braverman, On exponential stability of linear differential equations with several delays, Journal of Mathematical Analysis and Applications 324 (2006), no. 2, 1336-1355.

[3] _ On stability of some linear and nonlinear delay differential equations, Journal of Mathematical Analysis and Applications 314 (2006), no. 2, 391-411.

[4] T. Faria and W. Huang, Special solutions for linear functional differential equations and asymptotic behaviour, Differential and Integral Equations 18 (2005), no. 3, 337-360.

[5] S. A. Gusarenko and A. I. Domoshnitskiŭ, Asymptotic and oscillation properties of first-order linear scalar functional-differential equations, Differential Equations 25 (1989), no. 12, 1480-1491 (1990).

[6] I. Győri, Interaction between oscillations and global asymptotic stability in delay differential equations, Differential and Integral Equations 3 (1990), no. 1, 181-200.

[7] J. Hale, Theory of Functional Differential Equations, Springer, New York, 1977.

[8] M. Pituk, Convergence to equilibria in scalar nonquasimonotone functional differential equations, Journal of Differential Equations 193 (2003), no. 1, 95-130. 


\section{Exponential stability in FDEs}

[9] H. L. Smith, Monotone Dynamical Systems: An Introduction to the Theory of Competitive and Cooperative Systems, Mathematical Surveys and Monographs, vol. 41, American Mathematical Society, Rhode Island, 1995.

[10] H. L. Smith and H. R. Thieme, Monotone semiflows in scalar non-quasi-monotone functionaldifferential equations, Journal of Mathematical Analysis and Applications 150 (1990), no. 2, 289306.

Eduardo Liz: Departamento de Matemática Aplicada II, ETSI Telecomunicación,

Universidade de Vigo, Campus Marcosende, Vigo 36280, Spain

E-mail address: eliz@dma.uvigo.es

Mihály Pituk: Department of Mathematics and Computing, University of Veszprém,

P.O. Box 158, Veszprém 8201, Hungary

E-mail address: pitukm@almos.vein.hu 